\title{
卫星导航接收机抗欺骗干扰极大似然检测性能 分析
}

\author{
孙闽红*，王海泉*，张茴，沈雷 \\ 杭州电子科技大学通信工程学院, 杭州 310018 \\ * 通信作者. E-mail: cougar@hdu.edu.cn, tx_wang@hdu.edu.cn
}

收稿日期: 2013-08-19; 接受日期: 2013-10-31

国家自然科学基金 (批准号: 61271214) 和浙江省教育厅高校科研项目 (批准号: Y201121089) 资助

\begin{abstract}
摘要 欺骗千扰给卫星导航接收机带来巨大威胁, 而现有的文献均把欺骗千扰识别作为抗欺骗干 扰的主要研究方向, 而涉及欺骗干扰抑制方面的文献很少. 本文针对卫星导航接收机抗产生式欺骗 干扰问题, 根据接收机具有不同干扰先验信息的 3 种情形, 分别提出并证明了 3 个命题. 这些命题给 出了极大似然检测器在干扰背景下对真实卫星数据的检测性能界。命题表明, 在具备干扰信道先验 信息时, 极大似然检测器具有良好的抗产生式欺骗干扰性能. 仿真实验验证了理论分析的正确性和 有效性.
\end{abstract}

关键词卫星导航 接收机 抗干扰 欺骗干扰 极大似然检测

\section{1 引言}

针对卫星导航接收机的欺骗性干扰是干扰机发射与真实卫星导航信号相类似的干扰信号, 误导卫 星导航接收机偏离准确的导航和定位. 欺骗性干扰主要有转发式干扰和产生式干扰两种形式 ${ }^{[1,2]}$. 这 种干扰往往在时、频、空多域与卫星信号完全或部分重叠, 能够获得与真实信号相同的处理增益, 因而 所需干扰功率不大, 干扰效果较好. 文献 [3] 指出, 欺骗干扰功率只要高于真实信号 $4 \mathrm{~dB}$, 就可破坏典 型卫星导航接收机对真实信号的跟踪.

对于干扰抑制, 常规的滤波方法, 如空时域滤波 [4]、时频域滤波 [5] 都更适合于压制式干扰的抑制, 而用于抗欺骗干扰则往往效果不理想. 事实上, 卫星导航接收机抗欺骗的主要目标是准确地检测到欺 骗信号的存在, 并区分真实卫星信号和欺骗干扰信号. 在此基础上, 接收机可将接收信号中的欺骗信 号标记出来, 并排除在定时定位计算之外, 从而消除欺骗干扰对接收机的影响 [6]. 因而, 现有的研究均 集中在欺骗干扰的识别上 ${ }^{[7 \sim 13]}$. 目前公开的文献尚未见有对欺骗干扰抑制方法的研究.

产生式欺骗干扰的扩频序列、Doppler 频率、载波相位等信号参数尽可能与真实卫星信号一致, 并 附加上虚假的导航电文, 可以达到良好的欺骗效果. 本文针对产生式欺骗干扰抑制方法开展研究, 假定 接收机通过通信侦察获取了欺骗干扰的某些先验信息, 分析接收机采用极大似然法对接收数据进行解 码的性能界. 


\section{2 信号模型}

设卫星信号采用 BPSK 调制, 则接收信号中的真实卫星信号分量为

$$
s(t)=\sqrt{P_{s}} D(t) C(t) \cos \left[2 \pi\left(f_{0}+f_{d}\right) t+\theta_{s}\right],
$$

其中, $P_{s}$ 为受卫星信道衰落影响的信号功率, $D(t)$ 为数据码, $C(t)$ 为真实信号的扩频伪码, $f_{0}$ 与 $f_{d}$ 分 别为标称载波频率与载波多普勒频率, $\theta_{s}$ 为服从 $[0,2 \pi]$ 之间均匀分布的载波相位.

接收信号中的欺骗干扰分量为

$$
j(t)=\sqrt{P_{j}} D^{\prime}(t) C^{\prime}(t) \cos \left[2 \pi\left(f_{0}+f_{d}^{\prime}\right) t+\theta_{j}\right],
$$

其中, $P_{j}$ 为干扰信号功率且常有 $P_{j}>P_{s}, D^{\prime}(t)$ 为欺骗干扰产生的数据码, $C^{\prime}(t)$ 为欺骗干扰信号的扩 频伪码, $f_{d}^{\prime}$ 为干扰信号的多普勒频率, $\theta_{j}$ 为服从 $[0,2 \pi]$ 之间均匀分布的干扰信号载波相位. 产生式欺 骗干扰产生与真实卫星信号相同的扩频伪码与载波频率, 只是提供导航信息的数据码为错误码以干扰 用户的正常定位, 因而在接收机中可以获得与真实卫星信号相同的处理增益.

接收机接收到的信号是真实卫星信号、欺骗干扰信号与噪声三者的叠加, 即

$$
r(t)=s(t)+j(t)+n(t),
$$

其中, $s(t)$ 为式 (1) 的真实卫星信号, $j(t)$ 为式 (2) 的干扰信号, $n(t)$ 为加性高斯白噪声.

设卫星信道与干扰信道均为慢平坦衰落信道, 接收信号中的真实卫星信号与欺骗干扰信号均可被 顺利解扩和载波剥离, 则在某一时刻得到的基带信号模型为

$$
r=\sqrt{\rho_{s}} h_{s} x_{s}+\sqrt{\rho_{j}} h_{j} x_{j}+v,
$$

其中, $\rho_{s}$ 为信噪比 $(\mathrm{SNR}) ; \rho_{j}$ 为干扰与噪声比 $(\mathrm{JNR}) ; h_{s}$ 和 $h_{j}$ 分别为真实信号和欺骗干扰经历的瞬 时信道衰落, 假设它们都服从均值为 0 , 方差为 1 的高斯随机变量; $x_{s}$ 和 $x_{j}$ 分别为真实信号和欺骗干 扰的数据码; $v$ 为高斯白噪声, 且均值为 0 , 方差为 1 .

由于通常有 $\rho_{j}>\rho_{s}$, 此时直接对真实信号数据码进行解调将带来较高的误码率, 导致导航数据被 篡改. 基于此, 一种思路是采用 “估计 - 剔除” 法 [14] 1), 即先对更强的干扰信号进行估计, 然后从接 收信号中减去估计得到的干扰信号, 从而消除干扰. 从信息论角度已经证明, 当 $\rho_{j}$ 远大于 $\rho_{s}$ 时, 该方 法可以达到最优的性能 ${ }^{[14,15]}$. 然而, 从干扰机角度, 为了防止被侦察发现, 以保证自身安全和实现隐 蔽干扰的需要, 一般要求 $\rho_{j}$ 仅稍大于 $\rho_{s}$, 此时若继续采用 “估计 一 剔除” 的干扰抑制方法, 接收机 导航定位质量将仍然难以得到保证. 因此, 本文基于最大似然检测的思想, 寻找新的欺骗干扰抑制方 法. 另外, 通信系统中, 误码率或误符号率是根据信息准则来衡量干扰抑制效能的重要评价指标, 因而 本文以误码率/误符号率作为性能分析与比较的对象.

\section{3 极大似然检测及其性能分析}

设接收机已经检测识别出受到欺骗干扰, 通过侦察手段已经建立了干扰机的数据库, 从而具有干 扰机的先验信息. 实际中, 产生式欺骗干扰信号总是尽可能真地模仿真实卫星信号, 因而假定欺骗干扰

1) “估计 - 剔除” 法的主要步骤如下: 首先把 $\sqrt{\rho_{s}} h_{s} x_{s}+v$ 视作噪声从式 (4) 中解码出 $x_{j}$, 即 $\hat{x}_{j}=$ $\min _{x_{j} \in S}\left|r-\sqrt{\rho_{j}} h_{j} x_{j}\right|$, 其中 $S$ 为干扰用户所用的码本. 然后令 $\tilde{r}=r-\sqrt{\rho_{j}} h_{j} \hat{x}_{j}$, 再从 $\tilde{r}$ 中解码出 $x_{s}$, 即 $\hat{x}_{s}=$ $\min _{x_{s} \in S}\left|\tilde{r}-\sqrt{\rho_{s}} h_{s} x_{s}\right|$. 
与真实卫星信号的主要信号参数基本一致, 且只有导航电文数据不同, 但两者具有相同的码本是合理 的, 即可设定 $x_{s}, x_{j} \in S=\{-1,+1\}$. 根据接收机掌握先验信息的不同程度, 可以分为以下 3 种情形:

(1) 接收机已知卫星信道信息 $h_{s}$ 和干扰信道信息 $h_{j}$; 接收机可以同时解调出信息数据 $x_{s}$ 和 $x_{j}$.

(2) 接收机已知卫星信道信息 $h_{s}$ 和干扰信道信息 $h_{j}$; 接收机只解调出真实信号数据 $x_{s}$.

(3) 接收机已知卫星信道信息 $h_{s}$ 但未知干扰机到接收机的信道信息 $h_{j}$; 接收机将干扰视作高斯 噪声, 只能解调出真实信号数据 $x_{s}$.

以下分别对这 3 种情形进行讨论.

(1) 设接收机能够正确估计到 $h_{s}$ 和 $h_{j}$, 且干扰方使用与卫星真实信号相同的码本, 则联合解码极 大似然检测可以表示为

$$
\left(\hat{x}_{s 1}, \hat{x}_{j 1}\right)=\arg \min _{x_{s}, x_{j} \in S}\left\|r-\left(\sqrt{\rho_{s}} h_{s} x_{s}+\sqrt{\rho_{j}} h_{j} x_{j}\right)\right\|^{2} .
$$

可以证明, 该检测方法将优于前述的 “估计 一剔除” 法, 特别是在 $\rho_{s}$ 和 $\rho_{j}$ 相差不大的情况下.

实际上, 用户并不关心欺骗信息能否正确解调, 因而可以只计算真实导航信息的误码率.

命题 1 基于上述假设, 真实导航信息的成对误码率满足下式:

$$
P_{1} \leqslant \frac{1}{2 \sqrt{\rho_{s}+1}}+\frac{1}{2 \sqrt{1+\rho_{s}+\rho_{j}}} .
$$

该命题的证明见附录 A, B.

命题 1 给出了令人惊讶的结果: 随着干噪比 $\rho_{j}$ 的增加, 成对误码率将减小. 事实上, 这与 “估计 一剔除” 干扰抑制方法相吻合, 因为干扰越强, 干扰数据的估计越准确, 将干扰数据从接收数据中剔 除的干扰抑制性能越好.

(2) 设接收机能够正确估计到 $h_{s}$ 和 $h_{j}$, 且干扰方使用与卫星真实信号相同的码本, 但接收机只解 调出真实信号数据 $x_{s}$ 而不考虑欺骗数据 $x_{j}$ 的解调结果, 则极大似然检测器为

$$
\hat{x}_{s 1}=\arg \max _{x_{s} \in S} \sum_{x_{j} \in S} \exp \left(-\frac{\left\|r-\sqrt{\rho_{s}} h_{s} x_{s}\right\|^{2}}{2 \sigma^{2}}\right),
$$

式中 $\sigma^{2}$ 为 $\sqrt{\rho_{j}} h_{j} x_{j}+v$ 的方差. 对于这一情形, 有如下命题.

命题 2 基于上述假设, 真实导航信息的成对误码率满足

$$
P_{2} \leqslant P_{1} \leqslant \frac{1}{2 \sqrt{\rho_{s}+1}}+\frac{1}{2 \sqrt{1+\rho_{s}+\rho_{j}}} .
$$

该命题的证明见附录 C. 式 (8) 表明成对误码率将随着干扰的增强而减小, 且小于命题 1 中的成 对误码率.

(3) 接收机已知卫星信道信息 $h_{s}$ 但未知干扰机到接收机的信道信息 $h_{j}$, 接收机将干扰视作噪声, 则极大似然检测器可以表示为

$$
\hat{x}_{s 1}=\arg \max _{x_{s} \in \boldsymbol{S}} f\left(r \mid x_{s}, h_{s}\right) .
$$

此时, 有如下命题.

命题 3 基于上述假设, 真实导航信息的成对误码率满足下式

$$
P_{3} \leqslant \frac{1}{2} \sqrt{\frac{1+\rho_{j}}{1+\rho_{j}+\rho_{s}}} .
$$




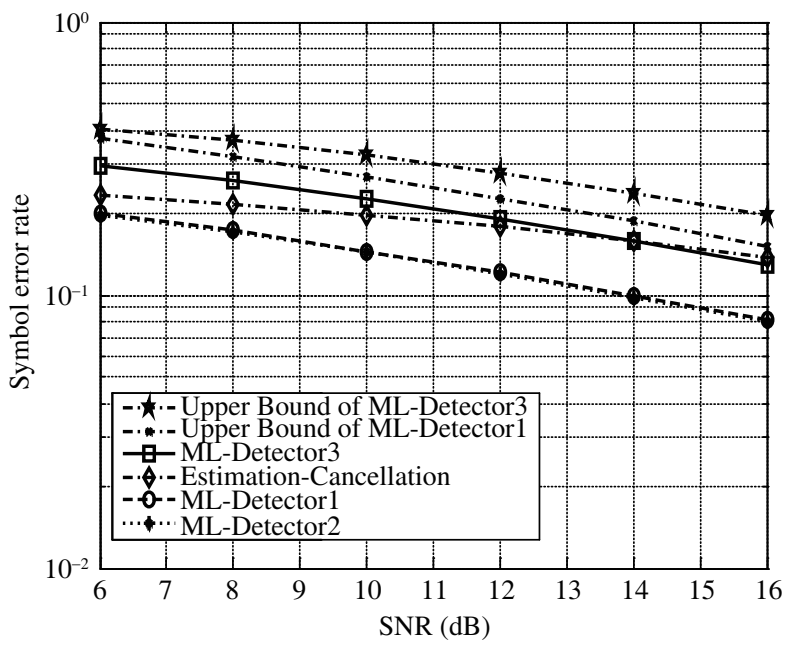

图 1 极大似然检测器与 “估计 - 剔除法” 误符号率性能比较 $(\mathrm{JNR}=8 \mathrm{~dB})$

Figure 1 Performance comparison between ML detectors and "estimation-cancellation" method ( $\mathrm{JNR}=8 \mathrm{~dB}$ )

该命题的证明见附录 D. 命题 3 表明, 如果接收机没有干扰信道先验信息, 成对误码率将随着干 扰的增强而增大.

上述 3 个命题中, 命题 3 中由于缺乏干扰的先验信息, 接收机无奈只能将干扰视作加性高斯噪声, 得到的检测性能必然是最差的, 这一情形也是目前接收机中常用的方法. 命题 1 与命题 2 均利用了干 扰的先验信息, 因而性能大大优于命题 3. 命题 1 中接收机未把干扰看作有害信息, 同时对真实数据 与欺骗数据同时解码, 运算复杂度较高; 命题 2 中只对真实数据解码而不考虑欺骗数据的解码正确与 否, 因而运算复杂度更低且性能更优.

\section{4 仿真实验}

分别设命题 1 至命题 3 中的极大似然检测器为 ML-Detector1、ML-Detector 2 和 ML-Detector3. $\mathrm{SNR}$ 定义为 $\mathrm{SNR} \triangleq 10 \log _{10}\left(P_{s} / \sigma^{2}\right)$, 这里 $P_{s}$ 与 $\sigma^{2}$ 分别为信号与噪声的平均功率. JNR 定义为 JNR $\triangleq 10 \log _{10}\left(P_{j} / \sigma^{2}\right)$, 这里 $P_{j}$ 为欺骗干扰的平均功率. 按照式 (4) 产生接收机信号, 并设定欺骗干扰信号 扩频码与多普勒频率等均与真实信号完全相同, 从而可以顺利实现解扩与载波剥离.

图 1 给出了指定 JNR 为 $8 \mathrm{~dB}, \mathrm{SNR}$ 以 $2 \mathrm{~dB}$ 为步长从 $6 \mathrm{~dB}$ 到 $16 \mathrm{~dB}$ 时 3 种极大似然检测器的 误符号率性能曲线. 同时, 为便于比较, 图中还给出了 “估计 - 剔除” 法的性能曲线以及分别由式 (6) 与式 (10) 确定的性能上界. 图 1 清晰表明, 4 种方法的误符号率由高到低依次为 ML-Detector 3、“估 计 - 剔除” 法、ML-Detector 1 和 ML-Detector 2. ML-Detector 3 与其上界相差约 $4 \mathrm{~dB}$, ML-Detector 1 与其上界相差约 $6 \mathrm{~dB}$, 但是理论上界与实际仿真曲线的下降斜率是一致的.

图 2 是指定 $\mathrm{SNR}$ 为 $8 \mathrm{~dB}$, JNR 以 $2 \mathrm{~dB}$ 为步长从 $6 \mathrm{~dB}$ 到 $16 \mathrm{~dB}$ 的仿真结果. 从图 2 也可以 看出, “估计 - 剔除” 法、ML-Detector 1 和 ML-Detector 2 的误符号率都随着 JNR 的增加而下降, 三 者中 “估计 - 剔除” 法的性能最差, ML-Detector 2 的性能最好, 且 ML-Detector 2 的误符号率略小于 ML-Detector 1, 这就分别验证了命题 1 与命题 2 中的式 (6) 和式 (8). 图 2 还表明, ML-Detector 3 的 误符号率随着 JNR 的增加而变大, 这就验证了命题 3 的式 (10).

以上仿真实验结果进一步验证了 3 个命题的正确性. 


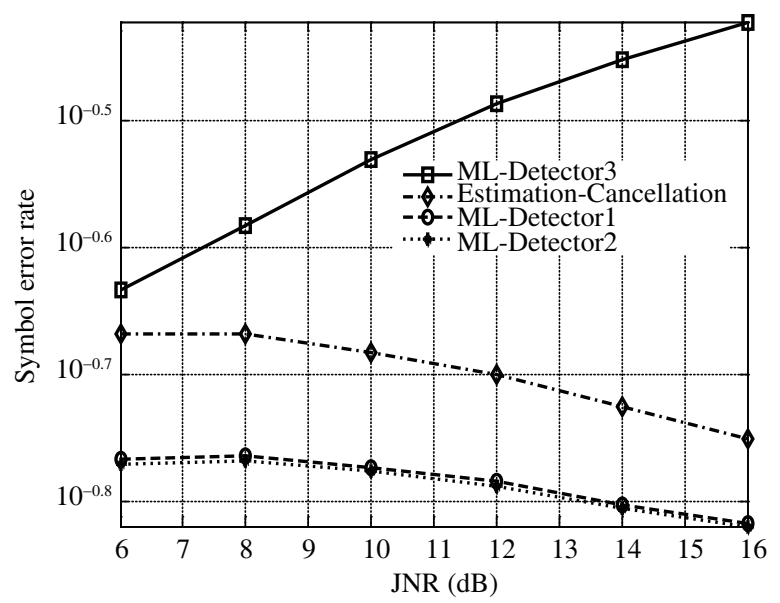

图 2 极大似然检测器与 “估计 - 剔除法” 误符号率性能比较 $(\mathrm{SNR}=8 \mathrm{~dB})$

Figure 2 Performance comparison between ML detectors and "estimation-cancellation" method (SNR=8 dB)

\section{5 结论}

本文针对卫星导航接收机受到产生式欺骗干扰这一情形, 理论分析并仿真验证了采用极大似然检 测器进行导航电文译码的性能. 理论分析结果表明: 如果缺乏干扰先验信息, 接收机在受到产生式欺 骗干扰时, 极大似然检测器的译码性能将随干扰功率的增强而变差; 而当接收机通过电子侦察等手段 获取了干扰信道信息时, 极大似然检测器的译码性能将随干扰功率的增强反而变好. 因此, 接收机若 能够借助通信侦察手段, 获取干扰信道的先验信息, 则接收机可采用文中命题 2 的检测方案, 即采用 极大似然检测器只对真实卫星数据解调而不考虑欺骗数据解调是否正确, 就能够实现干扰抑制, 且性 能随着干扰功率的增强反而变好. 这一结论为进一步研究接收机未知干扰信道信息时的抗欺骗干扰问 题打下基础.

\section{参考文献}

1 Volpe J A. Vulnerability Assessment of the Transportation Infrastructure Relying on the Global Positioning System. Wash-ington USA: National Transportation System Center, 2001

2 Scott L. Anti-spoofing \& authenticated signal architectures for civil navigation systems. In: Proceedings of the 16th International Technical Meeting of the Satellite Division of the Institute of Navigatio, Portland, 2003. 1543-1552

3 Huang L, Lü Z C, Wang F X. Spoofing pattern research on GNSS receivers. Astronaut, 2012, 33: 884-890 [黄龙, 吕志 成, 王飞雪. 针对卫星导航接收机的欺骗干扰研究. 宇航学报, 2012, 33: 884-890]

4 Niu F. Research on GNSS STAP interference suppression algorithm and its realization. Chengdu: University of Electronic and Science Technology, 2011 [牛飞. 导航接收机空时联合干扰抑制算法研究与实现. 成都: 电子科技大学, 2011]

5 Li Y G. Research on time-frequency anti-jamming techniques of GNSS and performance analysis. Chengdu: University of Electronic and Science Technology, 2011 [李玉广. 导航接收机时频抗干扰技术的研究和性能分析. 成都: 电子科技大学, 2011]

6 Huang L, Tang X M, Wang F X. Anti-spoofing techniques for GNSS receiver. Geomat Inf Sci Wuhan Univ, 2011, 36: $1344-1347$ [黄龙, 唐小妹, 王飞雪. 卫星导航接收机抗欺骗干扰方法研究. 武汉大学学报: 信息科学版, 2011, 36: 1344-1347]

7 Psiaki M L, O' Hanlon B W, Bhatti J A, et al. GPS Spoofing Detection via Dual-Receiver Correlation of Military Signals. IEEE Trans Aerosp Electron Syst, 2013, 49: 2250-2267 
8 Wullems C. A spoofing detection method for Civilian L1 GPS and the E1-B galileo safety of life service. IEEE Trans Aero Elec Sys, 2011, 48: 2849-2864

9 Nielsen J, Broumandan A, Lachapelle G. GNSS spoofing detection for single antenna handheld receivers. Navigation, 2011, 58: 335-344

10 Jafarnia-Jahromi A, Lin T, Broumandan A, et al. Detection and mitigation of spoofing attack on a vector based tracking GPS receiver. In: Proceedings of the International Technical Meeting of the Institute of Navigation, Newport Beach, 2012. 1-11

11 Cavaleri A, Motella B, Pini M, et al. Detection of spoofed GPS signals at code and carrier tracking level. In: Proceedings of the 5th ESA Workshop on Satellite Navigation Technologies and European Workshop on GNSS Signals and Signal Processing (NAVITEC), Noordwijk, 2010. 1-6

12 Gum A J, Rowitch D N. Anti-spoofing detection system. US patent, 20110227787A1, 2011

13 Nielsen J, Broumandan A, Lachapelle G. Method and system for detecting GNSS spoofing signals. US patent, 7952519, 2011

14 Carleial A. A case where interference does not reduce capacity. IEEE Trans Inform Theory, 1975, 21: 569-570

15 Sato H. The capacity of Gaussian interference channel under strong interference. IEEE Trans Inform Theory, 1981, 27: $786-788$

\section{附录 $\mathbf{A}$}

引理 设 $a$ 是一正常数, 则

$$
\frac{1}{\sqrt{2 \pi}} \int_{\|y\| \geqslant\|y-a\|} \exp \left(-\frac{\|y\|^{2}}{2}\right) \mathrm{d} y=Q\left(\frac{a}{2}\right)
$$

其中 $Q(\cdot)$ 为 $Q$ 函数.

证明 显然, 积分区域 $\|y\| \geqslant\|y-a\|$ 等价于 $\{y: y \geqslant a / 2\}$, 因此有

等式左边 $=\frac{1}{\sqrt{2 \pi}} \int_{y \geqslant a / 2} \exp \left(-\frac{\|y\|^{2}}{2}\right) \mathrm{d} y=Q\left(\frac{a}{2}\right)$, 上式最后的等式来自于 $Q$ 函数的定义.

\section{附录 B}

\section{命题 1 的证明}

显然, 成对误码率 $P_{1}$ 应为 $P_{1}=E\left[P_{1}\left(e \mid h_{s}, h_{j}\right)\right]$, 其中 $E[\cdot]$ 为求期望算子, $P_{1}\left(e \mid h_{s}, h_{j}\right)$ 为给定 $h_{s}$ 与 $h_{j}$ 条件 下的误码率. 若记 $P_{1}\left(x_{s 1} \rightarrow x_{s 2} \mid h_{s}, h_{j}\right)$ 为发送 $x_{s 1}$ 而被误判为 $x_{s 2}$ 的概率, 则有

$$
P_{1}\left(e \mid h_{s}, h_{j}\right)=P_{1}\left(x_{s 1} \rightarrow x_{s 2} \mid h_{s}, h_{j}\right) p\left(x_{s 1}\right)+P_{1}\left(x_{s 2} \rightarrow x_{s 1} \mid h_{s}, h_{j}\right) p\left(x_{s 2}\right),
$$

其中 $p\left(x_{s 1}\right)$ 和 $p\left(x_{s 2}\right)$ 分别为发送 $x_{s 1}$ 和 $x_{s 2}$ 的概率. 设两个码字等概率发送, 另由于 $x_{s 1}$ 和 $x_{s 2}$ 具有对称位置, 可 设 $P_{1}\left(x_{s 1} \rightarrow x_{s 2} \mid h_{s}, h_{j}\right)=P_{1}\left(x_{s 2} \rightarrow x_{s 1} \mid h_{s}, h_{j}\right)$, 因而有

$$
P_{1}\left(e \mid h_{s}, h_{j}\right)=P_{1}\left(x_{s 1} \rightarrow x_{s 2} \mid h_{s}, h_{j}\right) .
$$

现假设 $x_{s 1}$ 和 $x_{j 1}$ 被发送, 则正确事件发生的区域为

$$
\left\{r:\left\|r-d_{11}\right\| \leqslant\left\|r-d_{2 k}\right\|\right\} \cup\left\{r:\left\|r-d_{12}\right\| \leqslant\left\|r-d_{2 k}\right\|\right\},
$$

其中 $d_{i k}=\sqrt{\rho_{s}} h_{s} x_{s i}+\sqrt{\rho_{j}} h_{j} x_{j k}, i, k=1,2$. 因此, 导致错误的判决区域为

$$
D_{1}=\{w:\|w\| \geqslant\|w-a\| \text { or }\|w\| \geqslant\|w-a-b\|\} \cap\{w:\|w-b\| \geqslant\|w-a\| \text { or }\|w-b\| \geqslant\|w-a-b\|\},
$$

其中 $w \triangleq r-\sqrt{\rho_{s}} h_{s} x_{s 1}-\sqrt{\rho_{j}} h_{j} x_{j 1}, a \triangleq \sqrt{\rho_{s}} h_{s}\left(x_{s 2}-x_{s 1}\right), b \triangleq \sqrt{\rho_{j}} h_{j}\left(x_{j 2}-x_{j 1}\right)$. 因而有

$$
P_{1}\left(e \mid h_{s}, h_{j}\right)=\int_{D_{1}} f\left(r \mid h_{s}, h_{j}, x_{s 1}, x_{j 1}\right) \mathrm{d} r,
$$

其中 $f\left(r \mid h_{s}, h_{j}, x_{s 1}, x_{j 1}\right)$ 为给定 $h_{s}, h_{j}, x_{s 1}$ 和 $x_{j 1}$ 条件下接收信号 $r$ 的条件概率密度函数. 由假设与式 (4) 可知

$$
f\left(r \mid h_{s}, h_{j}, x_{s 1}, x_{j 1}\right)=\frac{1}{\sqrt{2 \pi}} \int_{D_{1}} \exp \left(-\frac{\|w\|^{2}}{2}\right) \mathrm{d} r .
$$


由于 $D_{1} \subseteq\{w:\|w\| \geqslant\|w-a\|$ or $\|w\| \geqslant\|w-a-b\|\}$, 有

$$
\begin{aligned}
P_{1}\left(e \mid h_{s}, h_{j}\right) & \leqslant \frac{1}{\sqrt{2 \pi}} \int_{\|w\| \geqslant\|w-a\|} \exp \left(-\frac{\|w\|^{2}}{2}\right) \mathrm{d} r+\frac{1}{\sqrt{2 \pi}} \int_{\|w\| \geqslant\|w-a-b\|} \exp \left(-\frac{\|w\|^{2}}{2}\right) \mathrm{d} r \\
& =Q\left(\left\|\frac{\sqrt{\rho_{s}}}{2} h_{s}\left(x_{s 2}-x_{s 1}\right)\right\|\right)+Q\left(\left\|\frac{\sqrt{\rho_{s}}}{2} h_{s}\left(x_{s 2}-x_{s 1}\right)+\frac{\sqrt{\rho_{j}}}{2} h_{j}\left(x_{j 2}-x_{j 1}\right)\right\|\right) .
\end{aligned}
$$

式中等式由前述引理得到. 利用不等式 $Q(x) \leqslant \exp \left(-x^{2} / 2\right) / 2$, 式 (B5) 可写为

$$
\begin{aligned}
P_{1}\left(e \mid h_{s}, h_{j}\right) \leqslant & \frac{1}{2} \exp \left(-\frac{1}{8} \rho_{s}\left\|h_{s}\left(x_{s 2}-x_{s 1}\right)\right\|^{2}\right) \\
& +\frac{1}{2} \exp \left(-\frac{1}{8}\left\|\sqrt{\rho_{s}} h_{s}\left(x_{s 2}-x_{s 1}\right)+\sqrt{\rho_{j}} h_{j}\left(x_{j 2}-x_{j 1}\right)\right\|^{2}\right) .
\end{aligned}
$$

对于式 (B6) 右边第 1 项, 由于 $h_{s} \sim N(0,1)$ 为服从高斯分布的实随机变量, 需对 $h_{s}$ 求统计平均, 有

$$
\begin{aligned}
& \frac{1}{\sqrt{2 \pi}} \int_{h_{s}} \frac{1}{2} \exp \left(-\frac{1}{8} \rho_{s}\left\|h_{s}\left(x_{s 2}-x_{s 1}\right)\right\|^{2}\right) \exp \left(-\frac{\left\|h_{s}\right\|^{2}}{2}\right) \mathrm{d} h_{s} \\
& =\frac{1}{2 \sqrt{2 \pi}} \int_{h_{s}} \exp \left[-\left(\frac{1}{8} \rho_{s}\left\|x_{s 2}-x_{s 1}\right\|^{2}+\frac{1}{2}\right)\left\|h_{s}\right\|^{2}\right] \mathrm{d} h_{s}=\frac{1}{\sqrt{\rho_{s}\left\|x_{s 2}-x_{s 1}\right\|^{2}+4}} .
\end{aligned}
$$

同理, 对式 (B6) 右边第 2 项, 令 $h=\sqrt{\rho_{s}} h_{s}\left(x_{s 2}-x_{s 1}\right)+\sqrt{\rho_{j}} h_{j}\left(x_{j 2}-x_{j 1}\right), h$ 服从均值为 0 , 方差 $\sigma^{2}=$ $\rho_{s}\left\|x_{s 2}-x_{s 1}\right\|^{2}+\rho_{j}\left\|x_{j 2}-x_{j 1}\right\|^{2}$ 的高斯分布. 对 $h$ 求统计平均有

$$
\begin{aligned}
& \frac{1}{\sqrt{2 \pi} \sigma} \int_{h} \frac{1}{2} \exp \left(-\frac{\|h\|^{2}}{8}\right) \exp \left(-\frac{\|h\|^{2}}{2 \sigma^{2}}\right) \mathrm{d} h \\
& =\frac{1}{2 \sqrt{2 \pi} \sigma} \int_{h} \exp \left[-\left(\frac{1}{8}+\frac{1}{2 \sigma^{2}}\right)\|h\|^{2}\right] \mathrm{d} h \\
& =\frac{1}{\sqrt{4+\sigma^{2}}}=\frac{1}{\sqrt{4+\rho_{s}\left\|x_{s 2}-x_{s 1}\right\|^{2}+\rho_{j}\left\|x_{j 2}-x_{j 1}\right\|^{2}}} .
\end{aligned}
$$

由于 $\left\|x_{s 2}-x_{s 1}\right\|^{2}$ 与 $\left\|x_{j 2}-x_{j 1}\right\|^{2}$ 等于 4 , 综合式 (B6) (B8), 可得

$$
P_{1} \leqslant \frac{1}{2 \sqrt{\rho_{s}+1}}+\frac{1}{2 \sqrt{1+\rho_{s}+\rho_{j}}} .
$$

\section{附录 $\mathrm{C}$}

\section{命题 2 的证明}

假设接收机已知 $x_{j} \in\{-1,+1\}$, 并设干扰信号发射 +1 和 -1 的概率相等, 则 $\sqrt{\rho_{j}} h_{j} x_{j}+v$ 服从的概率分布为

$$
\frac{1}{2} N\left(\sqrt{\rho_{j}} h_{j} x_{j 1}, 1\right)+\frac{1}{2} N\left(\sqrt{\rho_{j}} h_{j} x_{j 2}, 1\right) .
$$

此时, 接收信号 $r$ 服从的条件概率分布记为 $f\left(r \mid x_{s 1}, h_{s}, h_{j}\right)$, 且等于

$$
\frac{1}{2} N\left(\sqrt{\rho_{s}} h_{s} x_{s}+\sqrt{\rho_{j}} h_{j} x_{j 1}, 1\right)+\frac{1}{2} N\left(\sqrt{\rho_{s}} h_{s} x_{s}+\sqrt{\rho_{j}} h_{j} x_{j 2}, 1\right) .
$$

由式 (7) 可知, ML 检测器为

$$
\begin{aligned}
\hat{x}_{s} & =\arg \max _{x_{s} \in S} f\left(r \mid x_{s 1}, h_{s}, h_{j}\right) \\
& =\arg \max _{x_{s} \in S}\left[\frac{1}{2 \sqrt{2 \pi}} \mathrm{e}^{-\frac{\| r-\sqrt{\rho_{s} h_{s} x_{s 1}-\sqrt{\rho_{j}} h_{j} x_{j 1} \|^{2}}}{2}}+\frac{1}{2 \sqrt{2 \pi}} \mathrm{e}^{-\frac{\left\|r-\sqrt{\rho_{s}} h_{s} x_{s 1}-\sqrt{\rho_{j}} h_{j} x_{j 2}\right\|^{2}}{2}}\right] .
\end{aligned}
$$

1054 
成对误码率 $P_{2}$ 为

$$
\begin{aligned}
P_{2}\left(e \mid h_{s}, h_{j}\right) & =\int_{D_{2}} f\left(r \mid h_{s}, h_{j}, x_{s 1}\right) \mathrm{d} r \\
& =\frac{1}{2 \sqrt{2 \pi}} \int_{D_{2}}\left(\mathrm{e}^{-\frac{\left\|r-\sqrt{\rho s} h_{s} x_{s 1}-\sqrt{\rho_{j}} h_{j} x_{j 1}\right\|^{2}}{2}}+\mathrm{e}^{-\frac{\left\|r-\sqrt{\rho s} h_{s} x_{s 1}-\sqrt{\rho_{j}} h_{j} x_{j 2}\right\|^{2}}{2}}\right) \mathrm{d} r,
\end{aligned}
$$

其中

$$
\begin{aligned}
& D_{2}=\left\{r \mid\left[\mathrm{e}^{-\frac{\left(r-\sqrt{\rho_{s}} h_{s} x_{s 1}-\sqrt{\rho_{j}} h_{j} x_{j 1}\right)^{2}}{2}}+\mathrm{e}^{-\frac{\left(r-\sqrt{\rho_{s}} h_{s} x_{s 1}-\sqrt{\rho_{j}} h_{j} x_{j 2}\right)^{2}}{2}}\right]\right. \\
& \left.\leqslant\left[\mathrm{e}^{-\frac{\left(r-\sqrt{\rho_{s}} h_{s} x_{s 2}-\sqrt{\rho_{j}} h_{j} x_{j 1}\right)^{2}}{2}}+\mathrm{e}^{-\frac{\left(r-\sqrt{\rho_{s}} h_{s} x_{s 2}-\sqrt{\rho_{j}} h_{j} x_{j 2}\right)^{2}}{2}}\right]\right\}
\end{aligned}
$$

为错误决策区域. 对式 $(\mathrm{C} 4)$ 中的 $h_{s}$ 与 $h_{j}$ 求统计平均, 得到

$$
P_{2}=E_{h_{s}} E_{h_{j}}\left(\frac{1}{2 \sqrt{2 \pi}} \int_{D_{2}}\left[\mathrm{e}^{-\frac{\left\|r-\sqrt{\rho_{s} h_{s} x_{s 1}-\sqrt{\rho_{j}} h_{j} x_{j 1}}\right\|^{2}}{2}}+\mathrm{e}^{-\frac{\left\|r-\sqrt{\rho_{s} h_{s} x_{s 1}-\sqrt{\rho_{j}} h_{j} x_{j 2}}\right\|^{2}}{2}}\right] \mathrm{d} r\right) .
$$

容易证明有

$$
E_{h_{s}} E_{h_{j}}\left(\int_{D_{2}} \mathrm{e}^{-\frac{\| r-\sqrt{\rho s} h_{s} x_{s 1}-\sqrt{\rho_{j}} h_{j} x_{j 1}}{2} \|^{2}} \mathrm{~d} r\right)=E_{h_{s}} E_{h_{j}}\left(\int_{D_{2}} \mathrm{e}^{-\frac{\left\|r-\sqrt{\rho_{s} h_{s} x_{s 1}-\sqrt{\rho_{j}} h_{j} x_{j} 2}\right\|^{2}}{2}} \mathrm{~d} r\right) .
$$

因此, 有

$$
P_{2}=E_{h_{s}} E_{h_{j}}\left(\frac{1}{\sqrt{2 \pi}} \int_{D_{2}} \mathrm{e}^{-\frac{\left\|r-\sqrt{\rho s} h_{s} x_{s 1}-\sqrt{\rho_{j} h_{j} x_{j}}\right\|^{2}}{2}} \mathrm{~d} r\right) .
$$

接下来将 $P_{2}$ 与命题 1 中的 $P_{1}$ 进行比较. 首先定义两个集合, 一个是命题 1 中的正确决策区域 $\bar{D}_{1}$ 与错误决策 区域 $D_{2}$ 的交集, 定义为

$$
\begin{aligned}
D_{21} \triangleq \bar{D}_{1} \cap D_{2}= & \left\{\begin{array}{l}
\{w:\|w\| \leqslant\|w-a\| \text { and }\|w\| \leqslant\|w-a-b\|\} \cup \\
\{w:\|w-b\| \leqslant\|w-a\| \text { and }\|w-b\| \leqslant\|w-a-b\|\}
\end{array}\right\} \\
& \bigcap\left\{w: \mathrm{e}^{-\frac{\|w\|^{2}}{2}}+\mathrm{e}^{-\frac{\|w-b\|^{2}}{2}} \leqslant \mathrm{e}^{-\frac{\|w-a\|^{2}}{2}}+\mathrm{e}^{-\frac{\|w-a-b\|^{2}}{2}}\right\},
\end{aligned}
$$

这里的上划线表示集合的补集. 另一个是命题 1 中的错误决策区域 $D_{1}$ 与正确决策区域 $\bar{D}_{2}$ 的交集, 定义为

$$
\begin{aligned}
D_{22} \triangleq \bar{D}_{2} \cap D_{1}= & \left\{\begin{array}{l}
\{w:\|w\| \geqslant\|w-a\| \text { or }\|w\| \geqslant\|w-a-b\|\} \cup \\
\{w:\|w-b\| \geqslant\|w-a\| \text { or }\|w-b\| \geqslant\|w-a-b\|\}
\end{array}\right\} \\
& \bigcap\left\{w: \mathrm{e}^{-\frac{\|w-a\|^{2}}{2}}+\mathrm{e}^{-\frac{\|w-a-b\|^{2}}{2}} \leqslant \mathrm{e}^{-\frac{\|w\|^{2}}{2}}+\mathrm{e}^{-\frac{\|w-b\|^{2}}{2}}\right\} .
\end{aligned}
$$

那么 $P_{2}$ 与 $P_{1}$ 可以分别表示为

$$
\begin{aligned}
& P_{2}=\frac{1}{\sqrt{2 \pi}} E_{h_{s}} E_{h_{j}}\left(\int_{D_{2} \cap D_{1}} \mathrm{e}^{-\frac{\|w\|^{2}}{2}} \mathrm{~d} w\right)+\frac{1}{\sqrt{2 \pi}} E_{h_{s}} E_{h_{j}}\left(\int_{D_{21}} \mathrm{e}^{-\frac{\|w\|^{2}}{2}} \mathrm{~d} w\right), \\
& P_{1}=\frac{1}{\sqrt{2 \pi}} E_{h_{s}} E_{h_{j}}\left(\int_{D_{2} \cap D_{1}} \mathrm{e}^{-\frac{\|w\|^{2}}{2}} \mathrm{~d} w\right)+\frac{1}{\sqrt{2 \pi}} E_{h_{s}} E_{h_{j}}\left(\int_{D_{22}} \mathrm{e}^{-\frac{\|w\|^{2}}{2}} \mathrm{~d} w\right) .
\end{aligned}
$$

从上述两式可以看出, 要比较 $P_{2}$ 与 $P_{1}$ 的大小, 只要比较

$$
E_{h_{s}} E_{h_{j}}\left(\int_{D_{21}} \mathrm{e}^{-\frac{\|w\|^{2}}{2}} \mathrm{~d} w\right) \text { 与 } E_{h_{s}} E_{h_{j}}\left(\int_{D_{22}} \mathrm{e}^{-\frac{\|w\|^{2}}{2}} \mathrm{~d} w\right)
$$

的大小即可.

分别令

$$
A \triangleq\{w:\|w\| \leqslant\|w-a\|\},
$$




$$
\begin{gathered}
B \triangleq\{w:\|w\| \leqslant\|w-a-b\|\}, \\
C \triangleq\{w:\|w-b\| \leqslant\|w-a\|\}, \\
D \triangleq\{w:\|w-b\| \leqslant\|w-a-b\|\}, \\
E \triangleq\left\{w: \mathrm{e}^{-\frac{\|w\|^{2}}{2}}+\mathrm{e}^{-\frac{\|w-b\|^{2}}{2}} \leqslant \mathrm{e}^{-\frac{\|w-a\|^{2}}{2}}+\mathrm{e}^{-\frac{\|w-a-b\|^{2}}{2}}\right\},
\end{gathered}
$$

则 $D_{21}$ 可表示为 $D_{21}=\{(A \cap B) \cup(C \cap D)\} \cap E$. 容易证明

$$
\{(A \cap B) \cup(C \cap D)\} \cap E=\{(D \cup B) \cap(C \cup A)\} \cap E .
$$

作积分变换 $w=-z+a+b$, 则有

$$
\int_{D_{21}} \mathrm{e}^{-\frac{\|w\|^{2}}{2}} \mathrm{~d} w=\int_{\{(D \cup B) \cap(C \cup A)\} \cap E} \mathrm{e}^{-\frac{\|w\|^{2}}{2}} \mathrm{~d} w=\int_{\left\{\left(D^{\prime} \cup B^{\prime}\right) \cap\left(C^{\prime} \cup A^{\prime}\right)\right\} \cap E^{\prime}} \mathrm{e}^{-\frac{\|z-a-b\|^{2}}{2}} \mathrm{~d} z,
$$

其中, 集合相应变为

$$
\begin{gathered}
A \rightarrow A^{\prime} \triangleq\{z:\|z-a-b\| \leqslant\|z-b\|\}, \\
B \rightarrow B^{\prime} \triangleq\{z:\|z-a-b\| \leqslant\|z\|\}, \\
C \rightarrow C^{\prime} \triangleq\{z:\|z-a\| \leqslant\|z-b\|\}, \\
D \rightarrow D^{\prime} \triangleq\{z:\|z-a\| \leqslant\|z\|\}, \\
E \rightarrow E^{\prime} \triangleq\left\{z: \mathrm{e}^{-\frac{\|z-a-b\|^{2}}{2}}+\mathrm{e}^{-\frac{\|z-a\|^{2}}{2}} \leqslant \mathrm{e}^{-\frac{\|z-b\|^{2}}{2}}+\mathrm{e}^{-\frac{\|z\|^{2}}{2}}\right\} .
\end{gathered}
$$

由于 $\left\{\left(D^{\prime} \cup B^{\prime}\right) \cap\left(C^{\prime} \cup A^{\prime}\right)\right\} \cap E^{\prime}=D_{22}$, 有

$$
\int_{D_{21}} \mathrm{e}^{-\frac{\|w\|^{2}}{2}} \mathrm{~d} w=\int_{D_{22}} \mathrm{e}^{-\frac{\|w-a-b\|^{2}}{2}} \mathrm{~d} w .
$$

这样, 要比较 $P_{2}$ 与 $P_{1}$ 的大小, 变成只要比较

$$
E_{h_{s}} E_{h_{j}}\left(\int_{D_{22}} \mathrm{e}^{-\frac{\|w-a-b\|^{2}}{2}} \mathrm{~d} w\right) \text { 与 } E_{h_{s}} E_{h_{j}}\left(\int_{D_{22}} \mathrm{e}^{-\frac{\|w\|^{2}}{2}} \mathrm{~d} w\right)
$$

的大小了.

由集合运算法则, $D_{22}$ 可以展开为

$$
D_{22}=\left(D^{\prime} \cap C^{\prime} \cap E^{\prime}\right) \cup\left(B^{\prime} \cap C^{\prime} \cap E^{\prime}\right) \cup\left(D^{\prime} \cap A^{\prime} \cap E^{\prime}\right) \cup\left(B^{\prime} \cap A^{\prime} \cap E^{\prime}\right),
$$

并注意到 $D^{\prime} \cap A^{\prime} \cap E^{\prime}=\emptyset$ 和 $B^{\prime} \cap C^{\prime} \cap E^{\prime}=\emptyset$, 则

$$
D_{22}=\left(D^{\prime} \cap C^{\prime} \cap E^{\prime}\right) \cup\left(B^{\prime} \cap A^{\prime} \cap E^{\prime}\right) .
$$

由于 $\left(D^{\prime} \cap C^{\prime} \cap E^{\prime}\right) \cap\left(B^{\prime} \cap A^{\prime} \cap E^{\prime}\right)=\emptyset$, 因而有

$$
E_{h_{s}} E_{h_{j}}\left(\int_{D_{22}} \mathrm{e}^{-\frac{\|w-a-b\|^{2}}{2}} \mathrm{~d} w\right)=E_{h_{s}} E_{h_{j}}\left(\int_{D^{\prime} \cap C^{\prime} \cap E^{\prime}} \mathrm{e}^{-\frac{\|w-a-b\|^{2}}{2}} \mathrm{~d} w\right)+E_{h_{s}} E_{h_{j}}\left(\int_{B^{\prime} \cap A^{\prime} \cap E^{\prime}} \mathrm{e}^{-\frac{\|w-a-b\|^{2}}{2}} \mathrm{~d} w\right) .
$$

再做变量代换 $w=z+b$ 并做简单运算, 可发现

$$
E_{h_{s}} E_{h_{j}}\left(\int_{B^{\prime} \cap A^{\prime} \cap E^{\prime}} \mathrm{e}^{-\frac{\|w-a-b\|^{2}}{2}} \mathrm{~d} w\right)=E_{h_{s}} E_{h_{j}}\left(\int_{D^{\prime} \cap C^{\prime} \cap E^{\prime}} \mathrm{e}^{-\frac{\|w-a\|^{2}}{2}} \mathrm{~d} w\right) .
$$

从而, 式 (C14) 可以写为

$$
E_{h_{s}} E_{h_{j}}\left(\int_{D_{22}} \mathrm{e}^{-\frac{\|w-a-b\|^{2}}{2}} \mathrm{~d} w\right)=E_{h_{s}} E_{h_{j}}\left(\int_{D^{\prime} \cap C^{\prime} \cap E^{\prime}}\left(\mathrm{e}^{-\frac{\|w-a\|^{2}}{2}}+\mathrm{e}^{-\frac{\|w-a-b\|^{2}}{2}}\right) \mathrm{d} w\right) .
$$

同理, 可以得出

$$
E_{h_{s}} E_{h_{j}}\left(\int_{D_{22}} \mathrm{e}^{-\frac{\|w\|^{2}}{2}} \mathrm{~d} w\right)=E_{h_{s}} E_{h_{j}}\left(\int_{D^{\prime} \cap C^{\prime} \cap E^{\prime}}\left(\mathrm{e}^{-\frac{\|w\|^{2}}{2}}+\mathrm{e}^{-\frac{\|w-b\|^{2}}{2}}\right) \mathrm{d} w\right) .
$$


比较式 (C16) 与式 (C17), 并根据决策区域 $E$ 的条件, 可知

$$
E_{h_{s}} E_{h_{j}}\left(\int_{D_{22}} \mathrm{e}^{-\frac{\|w-a-b\|^{2}}{2}} \mathrm{~d} w\right) \leqslant E_{h_{s}} E_{h_{j}}\left(\int_{D_{22}} \mathrm{e}^{-\frac{\|w\|^{2}}{2}} \mathrm{~d} w\right) .
$$

由此可以得出结论

$$
P_{2} \leqslant P_{1} \leqslant \frac{1}{2 \sqrt{\rho_{s}+1}}+\frac{1}{2 \sqrt{1+\rho_{s}+\rho_{j}}} .
$$

\title{
附录 D
}

命题 3 的证明

接收机未知干扰信道信息 $h_{j}$ 且将干扰视作噪声. 设 $h_{j} \sim N(0,1)$, 则 $\sqrt{\rho_{j}} h_{j} x_{j}+v$ 是服从均值为 0 , 方差为 $\rho_{j}+1$ 的高斯分布.

成对误码率 $P_{3}$ 为

$$
P_{3}\left(e \mid h_{s}\right)=\int_{D_{3}} f\left(r \mid h_{s}, x_{s 1}\right) \mathrm{d} r=\frac{1}{\sqrt{2 \pi\left(1+\rho_{j}\right)}} \int_{D_{3}} \exp \left(-\frac{\left\|r-\sqrt{\rho_{s}} h_{s} x_{s 1}\right\|^{2}}{2\left(1+\rho_{j}\right)}\right) \mathrm{d} r,
$$

其中 $D_{3}=\left\{r \mid\left\|r-\sqrt{\rho_{s}} h_{s} x_{s 1}\right\|^{2} \geqslant\left\|r-\sqrt{\rho_{s}} h_{s} x_{s 2}\right\|^{2}\right\}$ 为错误决策区域. 进一步有

$$
P_{3}\left(e \mid h_{s}\right)=Q\left(\frac{\sqrt{\rho_{s}}\left\|h_{s}\left(x_{s 2}-x_{s 1}\right)\right\|}{2 \sqrt{1+\rho_{j}}}\right) .
$$

对式 (D2) 中的 $h_{s}$ 求统计平均, 得到

$$
\begin{aligned}
P_{3} & =\frac{1}{\sqrt{2 \pi}} \int Q\left(\frac{\sqrt{\rho_{s}}\left\|h_{s}\left(x_{s 2}-x_{s 1}\right)\right\|}{2 \sqrt{1+\rho_{j}}}\right) \mathrm{e}^{-\frac{\left\|h_{s}\right\|^{2}}{2}} \mathrm{~d} h_{s} \leqslant \frac{1}{2 \sqrt{2 \pi}} \int \exp \left(-\frac{\rho_{s}\left\|h_{s}\left(x_{s 2}-x_{s 1}\right)\right\|^{2}}{8\left(1+\rho_{j}\right)}-\frac{\left\|h_{s}\right\|^{2}}{2}\right) \mathrm{d} h_{s} \\
& =\sqrt{\frac{1+\rho_{j}}{\rho_{s}\left\|x_{s 2}-x_{s 1}\right\|^{2}+4\left(1+\rho_{j}\right)}}=\frac{1}{2} \sqrt{\frac{1+\rho_{j}}{1+\rho_{j}+\rho_{s}}} .
\end{aligned}
$$

\section{Performance analysis of maximum likelihood detection for satellite navigation receiver anti-spoofing}

\author{
SUN MinHong*, WANG HaiQuan*, ZHANG Hui \& SHEN Lei \\ School of Communication Engineering, Hangzhou Dianzi University, Hangzhou 310018, China \\ *E-mail: cougar@hdu.edu.cn, tx_wang@hdu.edu.cn
}

\begin{abstract}
Spoofing interference is a huge threat to satellite navigation receivers. Most existing works focus on identification of the spoofing interference, but research on the rejection of the spoofing is lacking. To make up this lack, in this paper, the rejection of the spoofing jamming is concerned. Specifically, three cases are discriminated according to situations of prior information on the spoofing at the receiver side. For each case, a maximum likelihood (ML) detector is designed and its performance is analyzed. These analyses show that the ML detectors can provide better anti-jamming performance when the receiver has prior information of the spoofing. Simulations confirm our theoretical analyses.
\end{abstract}

Keywords GNSS (global navigation satellite system), receiver, anti-jamming, spoofing jamming, ML detection 


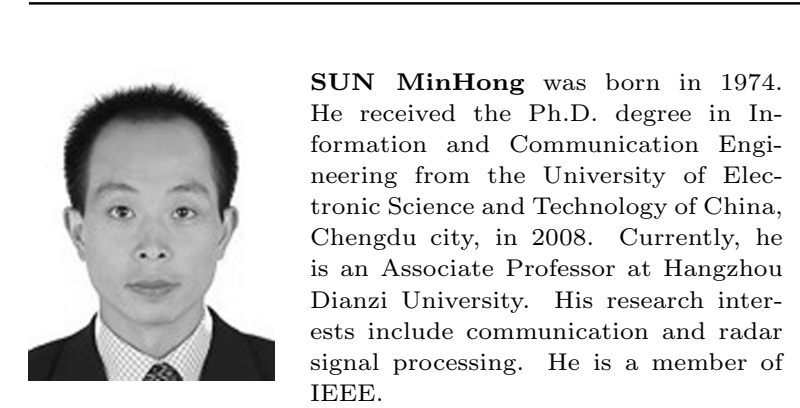

WANG HaiQuan was born in 1964 He received the Ph.D. degree in Mathematics from Kyoto Uni-versity, Japan, in 1997, and the Ph.D. degree in Electrical Engineering from University of Delaware, Newark, in 2005. Currently, he is a Professor at Hangzhou Dianzi University. His research interests include wireless communications, digital signal processing, and information theory. He is a member of IEEE. 